\title{
The Value of Virtual Chromoendoscopy in the Workup of Patients with Head and Neck Squamous Cell Carcinoma
}

\author{
Charlotte Van Lierde ${ }^{1,2}$ \& Brecht Gyselinck ${ }^{1,2}$ \& Jeroen Meulemans ${ }^{1,2}$ \& Raf Bisschops ${ }^{3}$ \& Pierre Delaere ${ }^{1}$ \& \\ Vincent Vander Poorten ${ }^{1,2}$
}

\begin{abstract}
Purpose of Review Second primary tumors (SPTs) significantly increase the mortality in patients with head and neck squamous cell carcinomas (HNSCCs). Virtual chromoendoscopy (VCE) could complement or replace lugol chromoendoscopy (LCE) for early esophageal second primary tumor (ESPT) detection. An overview of the existing techniques and their diagnostic performance in early detection of esophageal squamous cell neoplasms is provided.

Recent Findings Nowadays, LCE is the golden standard to detect ESPTs. Recently, multiple new VCE techniques have been developed. Especially narrow-band imaging (NBI) is promising. It shows similar sensitivity to LCE, but a significantly higher specificity.

Summary Patients with HNSCC are prone to develop ESPTs, both synchronous and metachronous, with a substantial negative impact on survival rates. Therefore, active screening and follow-up is necessary. LCE is an effective screening method, but has some disadvantages. Countering these drawbacks, NBI shows a high potential in early ESPT detection in high-risk patients. Additional multicenter studies are needed to compare diagnostic performance and cost-effectiveness of NBI and other VCE techniques with LCE.
\end{abstract}

Keywords Virtual chromoendoscopy $\cdot$ Lugol chromoendoscopy $\cdot$ Esophageal cancer · Second primary tumor $\cdot$ Head and neck cancer $\cdot$ Enhanced imaging

\section{Abbreviations}

AFI Autofluorescence imaging

BA Brownisharea

BLI Blue light imaging

DF-NBI Dual-focus narrow-band imaging

ESPT Esophageal second primary tumor

FICE Flexiblespectral imaging color enhancement

HNSCC Head and neck squamous cell carcinoma

HRME High-resolution microendoscopy

C. Van Lierde and B. Gyselinck are shared first authors.

This article is part of the Topical Collection on Head and Neck Cancers

* Vincent Vander Poorten

vincent.vanderpoorten@uzleuven.be

${ }^{1}$ Otorhinolaryngology - Head and Neck Surgery, Leuven Cancer Institute, University Hospitals Leuven, Herestraat 49, 3000 Leuven, Belgium

${ }^{2}$ Section Head and Neck Oncology, Department of Oncology, KU Leuven, Leuven, Belgium

${ }^{3}$ Gastroenterology, University Hospitals Leuven, Leuven, Belgium
iCLE Integrated confocallaser endoscopy

LCE Lugol chromoendoscopy

LVL Lugol voiding lesion

M Magnifying

NBI Narrow-band imaging

NM Non-magnifying

NPV Negative predictive value

pCLE Probe-based confocallaser endoscopy

PPV Positivepredictivevalue

SFORL Société Française d' Otorhinolaryngologie

SPT Second primary tumor

TNE Transnasal endoscopy

UADT Upper aerodigestive tract

VCE Virtual chromoendoscopy

WLE White light endoscopy

\section{Introduction}

The concept of field cancerization in patients with a primary head and neck squamous cell carcinoma (HNSCC) implies 
that exposure to carcinogens, such as alcohol and tobacco, induces the development of second primary tumors (SPTs) within the exposed mucosal membranes, throughout the entire upper aerodigestive tract (UADT) [1]. Thus, one quarter of all HNSCC patients will develop SPTs within 10 years after diagnosis [2]. One third of the mortality of patients with HNSCC is attributed to these neoplasms [2].

\section{Prevalence and Incidence of ESPT}

The overall prevalence of synchronous esophageal second primary tumors (ESPTs) in HNSCC patients is $5.1-28 \%$ [3, $4,5 \cdots, 6]$. This observed wide range is due to differences in patient characteristics, location of the index tumor, and the screening techniques that were used in the different studies [7]. The prevalence of synchronous ESPTs differs according to the site and stage of the primary tumor. Most ESPTs occur when the index tumor is located in the oral cavity and hypopharynx, with a prevalence up to $22 \%$ and $31 \%$ respectively $[8,9,10 \cdot]$.

A study with a 9-year follow-up of 9089 HNSCC patients observed an ESPT incidence of $9.4 \%$ over a period of 9 years; $42.1 \%$ were synchronous and $57.9 \%$ were metachronous $[5 \cdots]$.

\section{Survival Implications of ESPT}

ESPTs can be highly aggressive and bring 5-year survival rates down to $54.9 \%[11,12]$. One study observed a median overall survival of 38 months and a 5-year overall survival of $40 \%$ for patients with hypopharyngeal HNSCC [8]. In patients that were also diagnosed with an early or advanced stage synchronous ESPT, the median survival time was lowered to 36 and 15 months respectively [8]. However, when an ESPT is detected and treated early, the survival rate rises up to $80 \%$ $[11,13]$. Thus, an active screening program to detect ESPTs early increases survival rates significantly [14].

\section{Risk Factors for Developing ESPT}

Alcohol abuse (> $250 \mathrm{~g}$ of pure alcohol/day), smoking, lower age at diagnosis (median of $\leq 55$ years), and location of the index tumor (e.g., hypopharynx; a fortiori local invasion of $\geq$ 3 sites in the head and neck region) were found to increase the risk of developing a SPT [7, 8]. Even after treatment of the index HNSCC, the risk of developing a subsequent ESPT remains 8 to 22 times higher than in the general population $[3,7,15]$. This increased number of ESPTs is due to the carcinogenic effects of acetaldehyde, found in smoke and in the breakdown products of ethanol $[1,16]$. Two studies have shown that mainly alcohol consumption, but not smoking, implies this higher prevalence of synchronous ESPTs $[4,8]$. Another study, however, did identify smoking as an independent risk factor, while showing no effect of alcohol consumption on developing ESPTs [17]. These conflicting findings are due to the fact that alcohol and tobacco use are often closely linked and their effects difficult to evaluate separately [17]. Other identified risk factors for developing ESPTs are previous radiotherapy for breast cancer, caustic injury of the esophagus, achalasia, thylosis, drinking maté, chewing betelnut, exposure to polycyclic aromatic hydrocarbons, and race $[16,18]$. Logically, patients that develop other tobacco- and alcohol-related tumors like HNSCC and tracheobronchial cancer are considered high-risk patients for developing ESPTs [19, 20]. A lower BMI is an additional risk factor[21].

\section{Guidelines for Screening for ESPT in HNSCC Patients}

Early screening for ESPT in HNSCC patients is important, yet no global consensus on screening has been established [4]. National consensus documents do exist, and, e.g., in 2013 guidelines of the Société Française d' Otorhinolaryngologie (SFORL) recommended the use of lugol chromoendoscopy (LCE) in addition to white light endoscopy (WLE) in screening for ESPTs in patients with oro- and hypopharyngeal HNSCC and in patients with alcohol abuse [7]. After treating the index tumor, the annual risk of developing an ESPT remains 3-7\% [22]. Therefore, a routine screening for metachronous tumors during the follow-up is also indicated, and, thus, the 2015 SFORL guidelines propose that HNSCC patients be screened at 2-year intervals with LCE [22].

\section{Aim of This Study}

ESPTs are seen in 16-36\% of patients screened using the golden standard, LCE [17]. Virtual chromoendoscopy (VCE) is a group of relatively new endoscopic techniques developed for better, easier, and safer mucosal evaluation. Using VCE, ESPTs are potentially detected earlier, and earlier treatment of these could increase survival rates [14].

This study reviews the diagnostic value of the different VCE techniques in the detection of ESPTs in patients with HNSCC, and compares it with the standard of LCE.

\section{Materials and Methods}

A systematic review of the available chromoendoscopic techniques and their diagnostic performance was conducted. PubMed and Embase were searched using ("Head and Neck Neoplasms" [Mesh] AND "chromoendoscopy") for PubMed and ("head and neck tumor" AND "chromoendoscopy") for Embase. Articles published between January 1, 1990, and July 1, 2019, were included. This search led to 177 and 95 articles respectively. Afterwards, 8 articles were added by 
hand search. Only publications written in English and with an available full text were included. The study types of interest were systematic reviews, narrative reviews, randomized controlled trials, prospective studies, retrospective studies, cohort studies, and guidelines. Other inclusion criteria were human study objects, use of chromoendoscopy, synchronous or metachronous esophagus, and/or stomach neoplasms or dysplasia and patients with primary head and neck cancer. Only studies with numerical data on sensitivity, specificity, accuracy, PPV, and NPV were included. Studies on Barret's esophagus and case reports were excluded and no abstracts or data from unpublished research were accepted. Duplicate studies wereremoved.

Retrieved manuscripts were screened according to the PRISMA guidelines [23].

The flow diagram of the search process, using the PRISMA model, is outlined in Fig. 1 [23]. One hundred ninety-six abstracts did not meet the inclusion criteria. Of the remaining 84 articles, only 58 full texts could be obtained. These full texts were assessed for eligibility, including only the articles with numerical content on the diagnostic performances of the imaging techniques discussed in this paper. This resulted in 21 articles of which the data were collected and reviewed. The numerical data, such as sensitivity, specificity, diagnostic accuracy, area under sROC curve, positive predictive value (PPV), and negative predictive value (NPV), were extracted and summarized (Tables 1 and 2); their format, however, did not allow formulating the summary statistics.

\section{Results}

\section{Chromoendoscopy and Other Advanced Endoscopic Imaging Techniques}

Over the past 50 years, endoscopic imaging techniques have improved significantly. In the past, WLE was the golden standard to screen for ESPTs, but this technique was not very accurate in identifying intramucosal neoplasms such as erosions or flat mucosal lesions [30]. Recently, enhanced identification of suspicious mucosal lesions was observed with chromoendoscopy. This technique uses a dye sprayed on the mucosa to create a contrast between normal and neoplastic mucosa.

\section{Lugol Chromoendoscopy, the Current Golden Standard}

LCE is currently considered the golden standard to screen for ESPTs in high-risk patients. Normal glycogen-containing squamous epithelium absorbs lugol's iodine, whereas dysplastic, glycogen-depleted cells do not [39]. Due to the absence of lugol staining in malignant mucosa, visibility of suspicious mucosal lesions within the normal tissue is improved, and biopsies can be targeted to these lugol voiding lesions (LVLs) [5 $\cdot, 10 \cdot, 39]$. When screening HNSCC patients with LCE, 15\% are diagnosed with an ESPT, indicating both the high prevalence of SPTs as well as the usefulness of LCE to detect them $[10 \cdot]$. However, there are risks and side effects to the use of lugol, such as laryngospasm, chest pain, acute gastric mucosal damage, allergic reactions, pneumonitis, and nausea $[19,20,31$ * ]. LCE cannot be used in people with hyperthyroidism [29]. All these factors fed the search for safer methods for detecting ESPTs.

\section{New Advanced Endoscopic Imaging Techniques}

In VCE, the contrast to identify suspicious mucosal lesions is created by the endoscope itself, without using a dye. Enhancement of the real-time image can be optical or digital. Optical VCE uses optical filters in combination with high definition imaging incorporated in the light source of the endoscope [40, 41]. Digital VCE uses digital postprocessing algorithms to acquire enhanced images of the white light endoscopic image [40, 41]. Advantages of VCE include safe assessment of the upper third of the esophagus without causing chest discomfort, without the risk of allergic reaction to dyes or pulmonary aspiration [31 * ]. Disadvantages include training and less known diagnostic performances $[5 \cdots, 6,17]$.

1. Virtual chromoendoscopy with optical filters (preprocessing techniques)

\section{a. Narrow-band imaging (NBI)}

NBI is an optical VCE technique which is integrated with standard WLE in dedicated endoscopes. By the flick of a switch, the NBI setting filters the white light, leaving only blue and green light wavelengths [40]. These wavelengths uncover mucosal and submucosal vessel structures [40]. The blue 415-nm wavelength is absorbed by hemoglobin in mucosal vessels and the green 540 -nm wavelength is absorbed by the submucosal vessels [40]. Evaluating blood vessel structures helps in differentiating normal from suspicious lesions which show intrapapillary capillary loops [ 40 ]. Neovascularization and glycogen depletion lead to a brownish color of the mucosa when evaluating with NBI [ 5 • ]. These brownish areas (BAs) are considered suspicious and should be biopsied [31 • ]. A new technique, called dual-focus NBI (DF$\mathrm{NBI}$ ), was recently developed [42]. It creates an up to 80 times magnification of the NBI image, improving lesion evaluation.

\section{b. Blue light imaging (BLI)}

BLI is a quite new optical VCE technique. BLI is the blue mode of a four LED-light illumination source consisting of purple, green, red, and blue LEDs [29]. BLI's specific 
Table 1 Sensitivity, specificity, accuracy, area under sROC curve, PPV, NPV of imaging methods in detection of esophageal neoplasms in patients with primary head and neck cancer

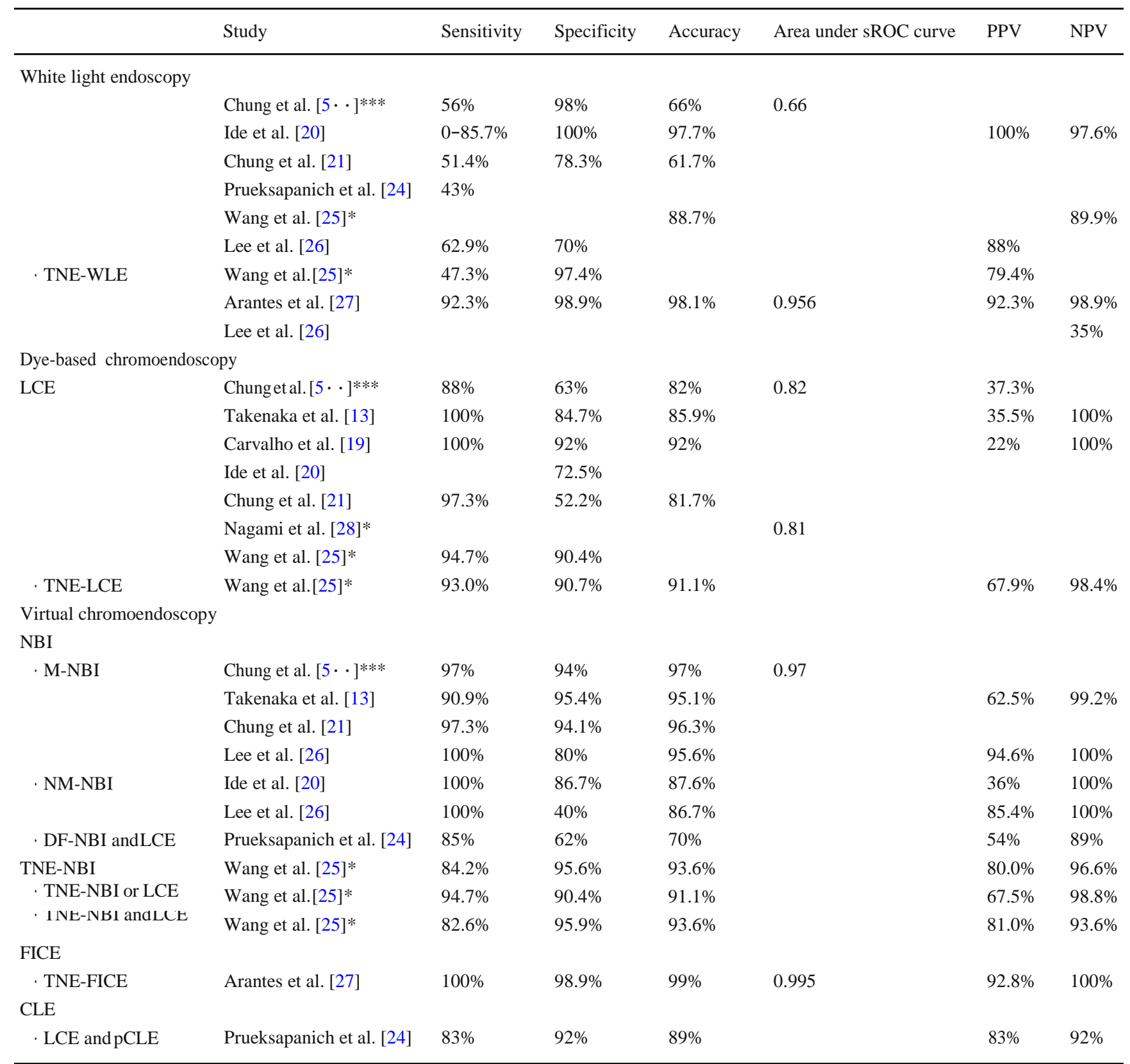

LCE lugol chromoendoscopy, NBI narrow-band imaging, $M$ magnifying, $N M$ non-magnifying, $D F-N B I$ dual-focus NBI, FICE flexible spectral imaging color enhancement, $C L E$ confocal laser endoscopy, $p C L E$ probe-based confocal laser endoscopy

*Prospective study $>200$ patients

***Systematic review

illumination light is used in combination withnon-magnifying NBI (NM-NBI) to improve the image of the latter [29]. It allows the endoscopic operator to choose between various light spectra when using NBI. The blue light spectrum increases visualization of mucosal patterns and superficial structures [29]. The distance at which lesions can be recognized as dysplastic or neoplastic is greater in BLI than in NBI, enabling better visualization of surrounding structures [21].

\section{c. Autofluorescence imaging (AFI)}

AFI uses the autofluorescent properties of endogenous fluorophores (NADH, collagen, and elastin) in the gastrointestinal tract [39]. These fluorophores emit longer wavelengths when excited with shorter wavelengths [39]. AFI uses a unique optical filter system with a blue excitation filter for inducing autofluorescence and a green filter for generating the 
Table 2 Sensitivity, specificity, accuracy, area under sROC curve, PPV, NPV of imaging methods in detection of esophageal neoplasms in high-risk patientsincluding HNSCCpatients

\begin{tabular}{|c|c|c|c|c|c|c|c|}
\hline & Study & Sensitivity & Specificity & Accuracy & Area under sROC curve & PPV & NPV \\
\hline \multicolumn{8}{|l|}{ White light endoscopy } \\
\hline & Diao et al. [29] & $66.7 \%$ & $42.3 \%$ & $56 \%$ & & & \\
\hline & Nagami et al. [28]* & $55.2-62.9 \%$ & & & & & \\
\hline \multicolumn{8}{|l|}{ Dye-based chromoendoscopy } \\
\hline \multirow[t]{8}{*}{ LCE } & Protano et al. [30] & $100 \%$ & $29 \%$ & $47 \%$ & $0.9559-0.9685$ & $32 \%$ & \\
\hline & Morita et al. $[31 \cdots]^{* * *}$ & $88-92 \%$ & $37-65 \%$ & & & & \\
\hline & Diao et al. [29] & $95.2 \%$ & $94.6 \%$ & $91.3 \%$ & & & \\
\hline & Huang et al. [32] & $89.1 \%$ & $90 \%$ & & & $97.6 \%$ & $64.3 \%$ \\
\hline & Nagami et al. [28]* & $94.2 \%$ & $64 \%$ & $68 \%$ & 0.80 & $28.6 \%$ & $98.6 \%$ \\
\hline & Christian et al. [33]* & $95.5 \%(\mathrm{M})$ & & & & & \\
\hline & Li et al. [34]* & $88.9 \%$ & & & & & \\
\hline & Li et al. [34]* & $92.6 \%(\mathrm{M})$ & & & & & \\
\hline . LCE and HRME & Protano et al. [30] & $95 \%$ & $79 \%$ & $83 \%$ & & $60 \%$ & \\
\hline \multirow[t]{2}{*}{$\cdot$ LCE-PS } & Goda et al. [35]** & $80.5 \%$ & $94.3 \%$ & $90.5 \%$ & & $84.6 \%$ & $92.6 \%$ \\
\hline & Ishihara et al. [36] & $88 \%$ & $95 \%$ & $91 \%$ & & $96 \%$ & $86 \%$ \\
\hline \multicolumn{8}{|l|}{ Virtual chromoendoscopy } \\
\hline \multicolumn{8}{|l|}{ NBI } \\
\hline \multirow[t]{2}{*}{ · NM-NBI and/or M-NBI } & Kandiah et al. [11] & & & $76.8-85.2 \%$ & & & \\
\hline & Morita et al. $[31 \cdots]^{* * *}$ & $94-98 \%$ & $82-88 \%$ & & $0.9587-0.9611$ & & \\
\hline \multirow[t]{3}{*}{$\cdot \mathrm{M}-\mathrm{NBI}$} & Diao et al. [29] & $95.2 \%$ & $92.8 \%$ & $87.5 \%$ & & & \\
\hline & Nagami et al. [28]* & $88.9-100 \%$ & & & & & \\
\hline & Goda et al. [35]** & $82.2 \%$ & $95.1 \%$ & $91.2 \%$ & & $88.1 \%$ & $92.4 \%$ \\
\hline • NM-NBI & Nagami et al. [28]* & $88.3 \%$ & $75.2 \%$ & $77.0 \%$ & 0.83 & $34.3 \%$ & $97.7 \%$ \\
\hline I-Scan & Guo et al. [37]* & $78.7 \%$ & $22.9 \%$ & $45.3 \%$ & & $40.7 \%$ & $61.5 \%$ \\
\hline I-Scan +pCLE & Guo et al. [37]* & $74.5 \%$ & $92.9 \%$ & $85.5 \%$ & & $87.5 \%$ & $84.4 \%$ \\
\hline \multicolumn{8}{|l|}{ CLE } \\
\hline \multirow[t]{2}{*}{ · LCE and pCLE } & Guo et al. [37]* & $94.6 \%$ & $90.7 \%$ & $92.3 \%$ & & $87.5 \%$ & $96.1 \%$ \\
\hline & Pech et al. [38] & & & $95 \%$ & & & \\
\hline$\cdot$ LCE andiCLE & Huang et al. [32] & $95.7 \%$ & $90.0 \%$ & $97.8 \%$ & & & \\
\hline \multicolumn{8}{|l|}{ FICE } \\
\hline$\cdot$ NM-FICE & Li et al. [34]* & $92.6 \%$ & & & & & \\
\hline$\cdot$ M-FICE & Li et al. [34]* & $96.3 \%$ & & & & & \\
\hline BLI & Diao et al. [29] & $95.2 \%$ & $91.9 \%$ & $85.7 \%$ & & & \\
\hline
\end{tabular}

$P P V$ positive predictive value, $N P V$ negative predictive value, $L C E$ lugol chromoendoscopy, $H R M E$ high-resolution magnifying endoscopy, $L C E-P S$ lugol chromoendoscopy pink stain sign, $N B I$ narrow-band imaging, $M$ magnifying, $N M$ non-magnifying, $C L E$ confocal laser endoscopy, $p C L E$ probebased confocal laser endoscopy, iCLE integrated confocal laser endoscopy, FICE flexible spectral imaging color enhancement, BLI blue light imaging *Prospective study $>200$ patients

***Rrandomized controlled trial

***Systematic review

reflected images. In conjunction with postprocessing algorithms of the generated images, a purple "red flag" lesion on AFI can be visualized and subsequently evaluated with highresolution endoscopy and NBI [39].

2. Virtual chromoendoscopy with digital filters (postprocessing techniques)

\section{a. Flexible spectral imaging color enhancement(FICE)}

FICE is a digital VCE technique. Here, a video processor disintegrates the endoscopic image into blue, green, and red components, which are then altered and resynthesized to compose a new image [39]. By focusing on different wavelengths and combining them, the contrast is enhanced and vascular 


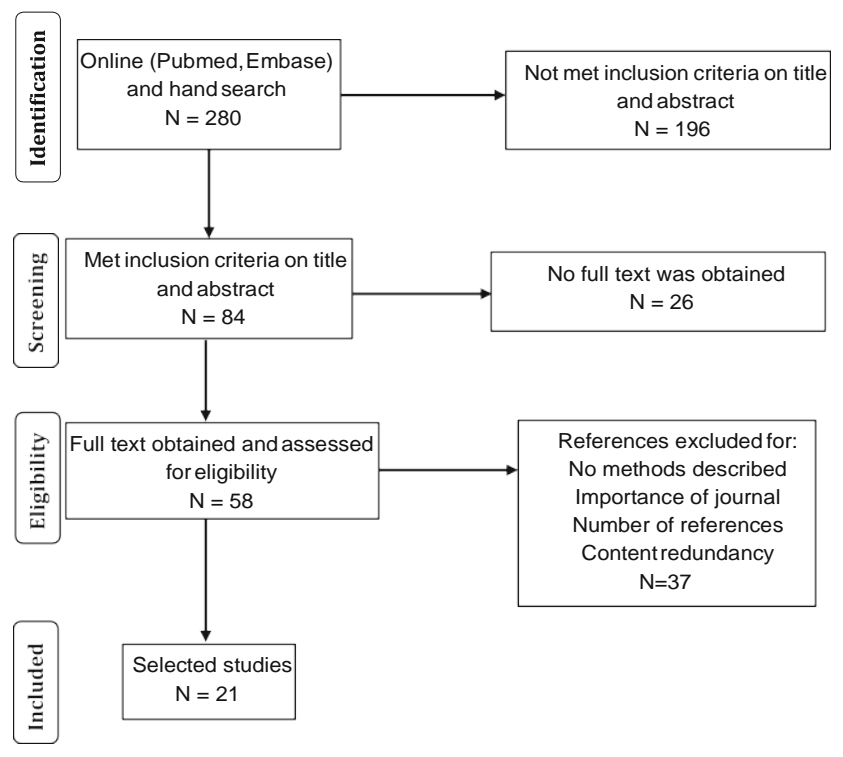

Fig. 1 Search strategy and study selection summarized in a flow diagram based on the PRISMA guidelines

patterns of the mucosa are highlighted [40]. One can choose between 10 different wavelength settings [40]. The image created by FICE, however, isa bit "noisier" in comparison to NBI.

\section{b. I-Scan}

I-Scan is a second digital VCE technique. It consists of a combination of three imaging algorithms: surface enhancement, tone enhancement, and contrast enhancement [37]. By adjusting the different settings, the identification of mucosal abnormalities can be improved [37, 40].

\section{Endoscopic microscopy}

a. Probe-based and integrated confocal laser endoscopy (pCLE andiCLE)

pCLE is a new optical VCE technique that provides real-life histological evaluation, whereas NBI and LCE require further biopsies [24, 32]. pCLE obtains a 1000-fold magnification with high-resolution images of the mucosal layer of the gastrointestinal tract, enabling cellular and intraepithelial papillary capillary loop evaluation [30, 32, 37, 39]. For good tissue contrast, intravenously injected fluorescein sodium is still needed [37]. CLE is very expensive ( $>\$ 150,000$ ) and only available in a few tertiary centers worldwide [30]. iCLE is a variant where the endoscope channel can still be used for lugol staining, while confocal images are simultaneously generated[32].

\section{b. High-resolution microendoscopy (HRME)}

HRME is a cheaper alternative for expensive techniques like CLE [30]. HRME is portable and battery operated, enabling its use in remote places and developing countries [30]. By inserting the $1 \mathrm{~mm}$ fiber-optic microendoscope in the accessory canal of the endoscope and applying the fluorescent proflavine, it allows delineation of cellular features, such as nuclear size, pleomorphism, and crowding [30]. Combined with LCE, it would increase specificity and decrease unnecessary biopsies [30]. Nowadays, HMRE is not commercially available yet [30].

\section{Summary of the Diagnostic Performance of Standard and Advanced Endoscopic Imaging Techniques}

The latest systematic reviews value LCE as a good technique to screen for ESPTs [ $\cdots, 31 \cdots$ ]. New VCE methods, however, show similar or better results with less risk and complications. Tables 1 and 2 give an overview of the reported diagnostic performance of these new techniques. Table 1 lists the data from studies focusing on patients with HNSCC. Table 2 does the same for studies that do not limit themselves to HNSCC, but also include other high-risk patients, such as patients with a history of esophageal squamous cell neoplasms, patients from high-risk countries, and patients with a score of $>6$ on the HRA-F health risk assessment model $[28,31 \cdots]$. In the literature, no studies evaluating the diagnostic performance of I-Scan, AFI, BLI, and HRME in HNSCC patients were found. Therefore, these techniques are excluded from Table 1. AFI has been excluded from Table 2 for the same reason in high-risk patients.

\section{Diagnostic Performance of Chromoendoscopy in Primary HNSCC Patients}

The diagnostic performances of WLE, LCE, NBI, TNE-NBI, FICE, and CLE for detecting ESPTs in HNSCC patients are displayed in Table 1. For LCE, one meta-analysis (16 studies, 4918 patients) found a mean sensitivity, specificity, accuracy, and area under the sROC curve of $88 \%, 63 \%, 82 \%$, and 0.82 respectively; in comparison, for NBI, the reported values were $97 \%, 94 \%, 97 \%$, and 0.97 respectively [ $\cdots$ ]. Area under the sROC curve of the respective techniques showed a higher diagnostic accuracy for NBI than for LCE [ 5 - ]. TNE-NBI can be combined with LCE. Its diagnostic performance varies depending on a parallel or serial approach. A parallel approach considers every BA on NBI or LVL on LCE as a positive lesion [25]. A serial approach only considers lesions that are BA on NBI and LVL on LCE as suspicious [25]. The parallel versus serial approach showed sensitivities of $94.7 \%$ versus $82.6 \%$. Specificities, on the other hand, where higher using the serial method or when using NBI alone [25], 95.9\% and 95.6\% respectively compared with $90.4 \%$ when using the parallel method [25]. One small study by Arantes et al. reported promising data for FICE, with a sensitivity, specificity, and accuracy of $100 \%$, 98.9\%, and 99\% [27]. 
Diagnostic Performance in High-Risk Patients Including HNSCC

The diagnostic performances of WLE, LCE, NBI, I-Scan, FICE, CLE, and BLI for detecting ESPT in high-risk patients are shown in Table 2. The literature shows similar sensitivities for LCE and NBI (80.5-100\% vs. 82.2-100\%) [28-36]. One study comparing both methods shows, for both techniques, a high area under the sROC curve (0.95-0.97) and thus a good sensitivity, without a significant difference [31 * ]. The same study, however, showed a significantly higher specificity when using NBI versus LCE (82-88\% vs. 37-65\%) [31 • ] ] Nagami et al. showed that, even for non-magnifying NBI (NM-NBI), the accuracy and specificity were significantly higher than with LCE (77\% vs. $68 \%$ and 75.2 vs. $64 \%, p=$ 0.03 and $p=0.01$ ) [28].

One prospective study comparing I-Scan and LCE concluded a non-inferiority in detection rate between the two techniques (10.4\% vs. $12.9 \%$, respectively) [37]. However, I-Scan showed an unsatisfactory screening performance with a sensitivity, specificity, and accuracy of $78.7 \%, 22.9 \%$, and $45.3 \%$ respectively [37]. The accuracy when combining IScan and pCLE increases significantly as compared with IScan alone (85.5\% vs. $45.3 \%, p<0.001)$ [37].

\section{Discussion}

Studies on Screening for ESPTs in HNSCC Patients-Discussion of the Data in Table 1

LCE is still considered as a reliable procedure for detecting ESPTs in HNSCC patients. The sensitivity of LCE and NBI is considered similar [ $\cdots$ - ]. However, the specificity of LCE (6392\%) is suboptimal as many non-neoplastic changes, such as atrophic change, create LVLs as well [5 • , 13, 19-21, 25]. Due to this non-specificity of LVLs, a lot of unnecessary biopsiesaretaken[5 $\cdots, 43]$.NBIand especially M-NBIshows a clearly higher specificity (80-95.9\%) [5 - , 13, 21, 26]. In accordance with our findings, Chung et al. concluded that NBI has a higher diagnostic performance and accuracy than WLE and LCE $[5 \cdot \cdots$. However, a limitation of their systematic review is the lack of a subgroup analysis between patients developing a synchronous and metachronous ESPT. A prospective study evaluating TNE-NBI and LCE, including 441 HNSCC patients, showed that the parallel approach of "NBI or LCE" achieved the highest diagnostic sensitivity (94.7\%) [25]. This parallel approach, where every positive lesion (BA on NBI or LVLs on LCE) was included, reduced the number of false negatives. Disadvantages of the parallel approach are longer procedure time, higher cost, and a higher complication rate [25]. In addition, this approach does not increase the specificity as compared with the use of TNE-NBI alone
[25]. Benefits of TNE in general are a higher completion rate (94-99\%), better passage of strictures, the absence of sedation, and shorter examination time (mean $17 \mathrm{~min}$ ) [25, 27]. Wang et al. considered TNE-NBI an effective screening method in detecting ESPTs in HNSCC patients [25].

Looking at NBI, both classical NM-NBI and M-NBI have been well studied. Like LCE, NM-NBI has a high sensitivity and negative predictive value (both up to $100 \%$, Table 1) [13, $19,20,26]$. No statistically significant difference in sensitivity between NM-NBI and M-NBI has been shown [26]. Nevertheless, specificity of M-NBI (80\%) is clearly higher than the specificity of NM-NBI (40\%), and this trend was observed by other authors resulting in a preference of $\mathrm{M}$ NBI over NM-NBI [26]. Lee et al. support the routine use of $\mathrm{NBI}$ as a screening technique in HNSCC patients, with the caveat that the diagnostic efficacy of NM-NBI vs. M-NBI remains somewhat ambiguous [26]. More prospective studies are needed to confirm these findings.

One prospective study, comprising 106 patients, showed that both WLE and FICE had a high accuracy in detecting and ruling out neoplastic lesions, but for ESPT detection, no significant difference in diagnostic performance between these two techniques was found [27]. This similar performance of FICE and WLE suggests that NBI and LCE are probably also superior to FICE, but formal comparative studies on the diagnostic differences between FICE, NBI, LCE, and other VCE techniques are needed to confirm this.

Studies on Screening for ESPTs in High-Risk Patients Including HNSCC Patients-Discussion of the Data in Table 2

First of all, it is important to note that the greater SPT prevalence in this high-risk population leads to a higher diagnostic yield (PPV) of the VCE techniques. A wide range of diagnostic performance for LCE was seen depending on the power, used methods, and the number of included patients. Overall, studies show no significant difference between the sensitivity of LCE and NBI [31 • ]. As mentioned before, LVLs are not very specific for ESPTs, leading to unnecessary biopsies $[31 \cdots]$. This fact implies a significant benefit for NBI, with its higher specificity [31 • $]$. Likewise, accuracy of NM-NBI has also shown to be superior to that of LCE, indicating the same potential for avoiding unnecessary biopsies [28]. NMNBI is widely used when no resources for magnification are available.

Theamount of irregular-shapedmultiformLVLspositively correlates with the risk of both synchronous and metachronous ESPT development [35, 44]. Takenaka et al. therefore recommended an initial LCE examination to assess the risk, and follow-up with NBI examinations to assess changes in mucosal vessel structures [13]. NBI still has other advantages. Unlike LCE, it is a push-on-a-button method 
without the use of a dye and therefore safer to use. NBI detects intrapapillary capillary loop patterns and thus a more exact assessment of diameter of lesions, as well as infiltration depth of neoplasms, influencing further treatment decisions [11, $31 \cdots$. Disadvantages include interobserver variability, the focused view (difficult to delineate lesions) and the absence of real-life histologic evaluation as seen in CLE. High NPVs for NBI are seen in less experienced as well as in experienced operators, $98 \%$ and $100 \%$ respectively, indicating that a patient without lesions on NBI can be spared further examination by LCE [45]. In patients with a lesion detected on NBI, Ishihara et al. still recommend the additional use of LCE, because of their belief in "its unsurpassed capacity to delineate malignant mucosal lesions" [45].

Few studies evaluating FICE, I-Scan and CLE have been conducted. FICE is considered complementary to LCE and has been shown to be a promising technique [27, 34, 37]. M-FICE and NM-FICE have shown similar detection rates as LCE [27, 34]. I-Scan too showed a non-inferiority when compared with LCE, despite its unsatisfactory screening performance (Table 2) [37]. I-Scan combined with pCLE did show a significant increase in overall accuracy [37]. Due to the narrow view, pCLE alone is not suited as a screening method. Nevertheless, pCLE is considered as an excellent method to differentiate between lesions that have been detected by other techniques. It can help target specific biopsies after previous lugol staining, significantly increasing specificity and thereby reducing the number of biopsies [24, 37]. Whether or not the iodine solution may lower image or video quality of pCLE is still uncertain [24, 37]. It also remains unclear whether to use pCLE before or after LCE, NBI, or IScan [43]. More studies are needed to compare VCE techniques and their combinations, in order to establish new cost-effective guidelines.

One small study evaluating BLI showed no significant difference in diagnostic performance between M-BLI, M-NBI, and LCE [29]. Larger multicenter studies evaluating BLI will still beneeded.

Limitations of the included studies in this review are that many studies struggle with selection bias and a confounded comparison of techniques, as the same operator performs the different VCE examinations. In addition, more than $70 \%$ of the included studies were conducted in Asian countries like Japan, China, and Taiwan. This finding correlated with the increased prevalence of head and neck cancer in these regions. One of the main reasons for this racial depending prevalence is the difference in the expression of the ALDH2 gene, which is responsible for the breakdown of acetaldehyde [16]. An inactive ALDH2 allele in the Asian population implies a higher level of acetaldehyde which has been linked to finding more suspicious LVLs [16]. Caucasians do not show polymorphisms in the ALDH2 gene [16]. Only a few European and US studies about this topic have been conducted, bearing the question whether theseinnovativetechniquesareequally costeffective in lower-risk regions.

\section{Conclusion}

Patients with HNSCC, especially of the hypopharynx, are prone to develop ESPTs, both synchronous and metachronous, with a substantial negative impact on survival rates. Therefore, active initial screening and follow-up is necessary. LCE is considered the golden standard for detecting ESPTs, but there are risks and side effects to the use of lugol $[19,20,31 \cdots]$. Of new VCE techniques, especially NBI constitutes an improvement in both HNSCC as in other high-risk patients, mainlybecause of a much higher specificity, decreasing the number of unnecessary biopsies, combined with avoidance of staining-related side effects. For now, I-Scan, FICE, and BLI do not show a significant improvement in diagnostic performance as compared with LCE. pCLE has the advantage that biopsies can be even better targeted due to instant histological evaluation, but availability is limited due to the high cost [30]. Future multicenter studies need to further evaluate and compare the diagnostic value and interobserver variability of the various VCE techniques, also focusing on cost-effectiveness andimplementation strategies.

\section{Compliance with Ethical Standards}

Conflict of Interest The authors declare that they have no conflicts of interest.

Human and Animal Rights and Informed Consent This article is based on previously conducted studies and does not contain any studies with human participants or animals performed by any of the authors. Ethical approval was provided by the Research Ethics Committee of the KU Leuven.

\section{References}

Papers of particular interest, published recently, have been highlighted as:

- Of importance

.. Of major importance

1. Slaughter DP, Southwick HW, SmejkalW. "Field cancerization" in oral stratified squamous epithelium. Clinical implications of multicentric origin. Cancer. John Wiley \& Sons, Ltd; 1953 [cited 2019 May 14];6:963-8. Available from: http://doi.wiley.com/10. 1002/1097-0142\%28195309\%296\%3A5\%3C963\%3A\%3AAIDCNCR2820060515\%3E3.0.CO\%3B2-Q

2. Lin K, Patel SG, Chu PY, Matsuo JMS, Singh B, Wong RJ, et al. Second primary malignancy of the aerodigestive tract in patients treated for cancer of the oral cavity and larynx. Head Neck. John Wiley \& Sons, Ltd; 2005 [cited 2019 May 14];27:1042-8. Available from: http://doi.wiley.com/10.1002/hed.20272 
3. Chung CS, Lee YC, Wang CP, Ko JY, Wang WL, Wu MS, et al. Secondary prevention of esophageal squamous cell carcinoma in areas where smoking, alcohol, and betel quid chewing are prevalent. J Formos Med Assoc. Singapore. 2010;109:408-21.

4. Wang YK, Chuang YS, Wu TS, Lee KW, Wu CW, Wang HC, et al. Endoscopic screening for synchronous esophageal neoplasia amongpatients with incidenthead andneck cancer:prevalence, risk factors, and outcomes. Int J Cancer. I.-C. Wu, Division of Gastroenterology, Department of Internal Medicine, Kaohsiung Medical University Hospital, Taiwan, United States: John Wiley \& Sons, Ltd; 2017 [cited 2019 Feb 11];141:1987-96. Available from: http://www.embase.com/search/results?subaction= viewrecord\&from=export\&id=L618371030\%0A https://doi.org/ 10.1002/ijc.30911

5. - Chung CS, Lo WC, Lee YC, Wu MS, Wang HP, Liao LJ. Imageenhanced endoscopy for detection of second primary neoplasm in patients with esophageal and head and neck cancer: a systematic review and meta-analysis. Eisele DW, editor. Head Neck. L.-J. Liao, Department of Otolaryngology, Far Eastern Memorial Hospital, No. 21, Nan-Ya South Road, Banciao District, New Taipei City, Taiwan; 2016;38:E2343-9. This is a recent systematic review focused on detecting ESPTs in patients with HNSCC. This study compares WLE, LCE, and NBI. The study showed NBI to have the best diagnostic performance in detecting ESPTs.

6. Möschler O, Spahn TW, Middelberg-Bisping C, Grosse-Thie W, Christoph B, Kloeppel G, et al. Chromoendoscopy is a valuable tool for screening of high-risk patients with head and neck cancer for early detection of esophageal cancer. Digestion. Switzerland. 2006;73:160-6.

7. De Monès E, Bertolus C, Salaun PY, Dubrulle F, Ferrié JC, Temam $S$, et al. Initial staging of squamous cell carcinoma of the oral cavity, larynx and pharynx (excluding nasopharynx). Part 2: Remote extension assessment and exploration for secondary synchronous locations outside of the upper aerodigestive tract. 2012 SFORL guidelines. Eur Ann Otorhinolaryngol Head Neck Dis. 2013 [cited 2019 Jul 18];130:107-12. Available from: https://linkinghub. elsevier.com/retrieve/pii/S1879729612001202

8. Ni XG, Zhang QQ, Zhu JQ, Wang GQ. Hypopharyngeal cancer associated with synchronous oesophageal cancer: risk factors and benefits of image-enhanced endoscopic screening. J Laryngol Otol. X.-G. Ni, Department of Endoscopy, National Cancer Center/ Cancer Hospital, Chinese Academy of Medical Sciences and Peking Union Medical College, No. 17 Panjiayuannanli, Chaoyang District PO Box 2258, Beijing, China, England; 2018;132:154-61. Available from: http://www.embase.com/ search/results? subaction=viewrecord \&from=export\&id= L620782889\%0A https://doi.org/10.1017/S0022215117002493

9. Matsui T, Okada T, Kawada K, Okuda M, Ogo T, Nakajima Y, et al. Detection of second primary malignancies of the esophagus and hypophraynx in oral squamous cell carcinoma patients. Laryngoscope Investig Otolaryngol. T. Okada, Department of Gastrointestinal Surgery, Tokyo Medical and Dental University, Tokyo, Japan: Wiley-Blackwell; 2018 [cited 2019 Jun 19];3:2637. Available from: http://www.embase.com/search/results? subaction=viewrecord\&from=export\&id=L623749897

10. Bugter O, van de Ven SEM, Hardillo JA, Bruno MJ, Koch AD, Baatenburg de Jong RJ. Early detection of esophageal second primary tumors using lugol chromoendoscopy in patients with head and neck cancer: a systematic review and meta-analysis. Head Neck. John Wiley \& Sons, Ltd; 2019 [cited 2019 Jun 19];41: 1122-30. Available from: https://onlinelibrary.wiley.com/doi/abs/ 10.1002/hed.25548 This is a recent published systematic review about the detection rate of ESPTs in patients with HNSCC when screened with LCE. Up to $15 \%$ of the HNSCC patients screened with LCE were diagnosed with an ESPT, showing the importance of endoscopic screening.

11. Kandiah K, Chedgy FJQ, Subramaniam S, Thayalasekaran S, Kurup A, Bhandari P. Early squamous neoplasia of the esophagus: the endoscopic approach to diagnosis and management. Saudi J Gastroenterol. India: Wolters Kluwer - Medknow Publications; 2017 [cited 2019 Jun 19];23:75-81. Available from: http://www. ncbi.nlm.nih.gov/pubmed/28361837.

12. Khoshbaten M, Naderpour M, Mohammadi G, Alipoor SH, Estakhri R, Fazeli Z. Epidemiology of esophageal lesions in patients with head and neck squamous cell carcinoma. Asian Pac J Cancer Prev. Thailand; 2010;11:863-5. Available from: http:// www.ncbi.nlm.nih.gov/pubmed/21133592.

13. Takenaka R, Kawahara Y, Okada H, Hori K, Inoue M, Kawano S, et al. Narrow-band imaging provides reliable screening for esophageal malignancy in patients with head and neck cancers. Am J Gastroenterol. 2009 [cited 2019 Feb 11];104:2942-8. Available from: http://www.nature.com/articles/ajg2009426

14. Wang WL, Wang CP, Wang HP, Lee CT, Chang CY, Tai CM, et al. The benefit of pretreatment esophageal screening with image-enhanced endoscopy on the survival of patients with hypopharyngeal cancer. Oral Oncol. 2013 [cited 2019 Jul 18];49: 808-13. Available from: https://doi.org/10.1016/j.oraloncology. 2013.04.009

15. Lim H, Kim DH, Jung HY, Gong EJ, Na HK, Ahn JY, et al. Clinical significance of early detection of esophageal cancer in patients with head and neck cancer. Gut Liver. 2015 [cited 2019 Feb 11];9:159-66. Available from: http://www.gutnliver.org/ journal/DOIx.php?id=10.5009/gnl13401

16. Muto M. Association between aldehyde dehydrogenase gene polymorphisms and the phenomenon of field cancerization in patients with head and neck cancer. Carcinogenesis. England; 2002;23: 1759-66. Available from: http://www.ncbi.nlm.nih.gov/pubmed/ 12376487.

17. Kim DH, Gong EJ, Jung HY, Lim H, Ahn JY, Choi KS, et al. Clinical significance of intensive endoscopic screening for synchronous esophageal neoplasm in patients with head and neck squamous cell carcinoma. Scand J Gastroenterol. Taylor \& Francis; 2014 [cited 2019 Feb 11];49:1486-92. Available from: http:// www.embase.com/search/results?subaction=viewrecord\&from= export\&id=L605260400.

18. Lopes AB. Esophageal squamous cell carcinoma - precursor lesions and early diagnosis. World J Gastrointest Endosc. Baishideng Publishing Group Inc; 2012 [cited 2019 Jul 21];4:9. Available from: http://www.ncbi.nlm.nih.gov/pubmed/22267978.

19. Carvalho R, Areia M, Brito D, Saraiva S, Alves S, Cadime AT. Diagnostic accuracy of lugol chromoendoscopy in the oesophagus in patients with head and neck cancer. Rev Esp Enfermedades Dig. R. Carvalho, Gastroenterology Unit, Coimbra Portuguese Institute of Oncology, Avenida Bissaya Barreto, 98, Apartado 2005, 3001651 Coimbra, Portugal: SociedadEspañola dePatología Digestiva/ Arán Ediciones, S.L.; 2013;105:79-83.

20. Ide E, Maluf-Filho F, Chaves DM, Matuguma SE, Sakai P. Narrow-band imaging without magnifcation for detecting early esophageal squamous cell carcinoma. World J Gastroenterol. E. Ide, University of São Paulo, Gastrointestinal Endoscopy Unit, São Paulo 05403-900, Brazil;2011;17:4408-13.

21. Chung CS, Liao LJ, Lo WC, Chou YH, Chang YC, Lin YC, et al. Risk factors for second primary neoplasia of esophagus in newly diagnosed head and neck cancer patients: a case-control study. BMC Gastroenterol. T.-H. Lee, Departments of Internal Medicine, Division of Gastroenterology and Hepatology, Far Eastern Memorial Hospital, No. 21, Nan-Ya South Road, Section 2, Ban-Ciao, 22060 New Taipei City, Taiwan: BioMed Central; 2013 [cited 2019 Jun 19];13:154. Available from: http:// 
www.embase.com/search/results?subaction=viewrecord\&from= export\&id=L52839406.

22. Blanchard D, Barry B, De Raucourt D, Choussy O, Dessard-Diana B, Hans S, et al. Guidelines update: post-treatment follow-up of adult head and neck squamous cell carcinoma: screening for metastasis and metachronous esophageal and bronchial locations. Eur Ann Otorhinolaryngol Head Neck Dis. 2015 [cited 2019 Jul 18];132:217-21. Available from: https://doi.org/10.1016/j. anorl.2015.07.001

23. Pati D, Lorusso LN. How to write a systematic review of the literature. Heal Environ Res Des J. 2018;11:15-30.

24. Prueksapanich P, Pittayanon R, Rerknimitr R, Wisedopas N, Kullavanijaya $\mathrm{P}$. Value of probe-based confocal laser endomicroscopy (pCLE) and dual focus narrow-band imaging (dNBI) in diagnosing early squamous cell neoplasms in esophageal lugol's voiding lesions. Endosc Int Open. R. Rerknimitr, Division of Gastroenterology, Department of Medicine, Faculty of Medicine, Chulalongkorn University, Bangkok, Thailand; 2015;03:E281-8. Available from: http://www.embase.com/ search/results?subaction=viewrecord \&from=export\&id= L610670860.

25. Wang CH, Lee YC, Wang CP, Chen CC, Ko JY, Han ML, et al. Use of transnasal endoscopy for screening of esophageal squamous cell carcinoma in high-risk patients: yield rate, completion rate, and safety. Dig Endosc Australia. 2014;26:24-31.

26. Lee CT, Chang CY, Lee YC, Tai CM, Wang WL, Tseng PH, et al. Narrow-band imaging with magnifying endoscopy for the screening of esophageal cancer in patients with primary head and neck cancers. Endoscopy. 2010 [cited 2019 Aug 31];42:613-9. Available from: http://www.ncbi.nlm.nih.gov/pubmed/20669074.

27. Arantes V, Albuquerque W, Salles JMP, Freitas Dias CA, Alberti LR, Kahaleh M, et al. Effectiveness of unsedated transnasal endoscopy with white-light, flexible spectral imaging color enhancement, and lugol staining for esophageal cancer screening in high-risk patients. J Clin Gastroenterol United States. 2013;47:314-21.

28. Nagami $Y$, Tominaga K, Machida H, Nakatani M, Kameda N, Sugimori S, et al. Usefulness of non-magnifying narrow-band imaging in screening of early esophageal squamous cell carcinoma: a prospective comparative study using propensity score matching. Am J Gastroenterol United States. 2014;109:845-54.

29. Diao W, Huang X, Shen L, Zeng Z. Diagnostic ability of blue laser imaging combined with magnifying endoscopy for early esophageal cancer. Dig Liver Dis Netherlands. 2018;50:1035-40.

30. Protano MA, Xu H, Wang G, Polydorides AD, Dawsey SM, Cui J, et al.Low-costhigh-resolution microendoscopy for the detection of esophageal squamous cell neoplasia: an international trial. Gastroenterol United States. 2015;149:321-9.

31. . Morita FHA, Bernardo WM, Ide E, Rocha RSP, Aquino JCM, Minata MK, et al. Narrow band imaging versus lugol chromoendoscopy to diagnose squamous cell carcinoma of the esophagus: a systematic review and meta-analysis. BMC Cancer. England. 2017:17-54 This is a recent systematic review focused on detecting ESPTs. The study compares LCE and NBI. NBI showed to be a good tool in the diagnosis of ESPTs. NBI performs even better than LCEin differentiating between different types of lesions.

32. Huang J, Yang YS, Lu ZS, Wang SF, Yang J, Yuan J. Detection of superficial esophageal squamous cell neoplasia by chromoendoscopy-guided confocal laser endomicroscopy. World J Gastroenterol United States. 2015;21:6974-81.

33. Christian W. TNM Atlas. 6th editio. Asamura H. SLH, editor. TNM Atlas. Wiley-Blackwell; 2014. Available from: https://www.wiley. com/en-be/TNM+Atlas\%2C+6th+Edition-p-9781118695647.
34. Li YX, Shen L, Yu HG, Luo HS, Yu JP. Fujinon intelligent color enhancement for the diagnosis of early esophageal squamous cell carcinoma and precancerous lesion. Turkish J Gastroenterol Turkey. 2014;25:365-9.

35. Goda K, Dobashi A, Yoshimura N, Kato M, Aihara H, Sumiyama $K$, et al. Narrow-band imaging magnifying endoscopy versus lugol chromoendoscopy with pink-color sign assessment in the diagnosis of superficial esophageal squamous neoplasms: a randomised noninferiority trial. Gastroenterol Res Pract. 2015;2015:1-10.

36. Ishihara R, Yamada T, Iishi H, Kato M, Yamamoto S, Yamamoto $\mathrm{S}$, et al. Quantitative analysis of the color change after iodine staining for diagnosing esophageal high-grade intraepithelial neoplasia and invasive cancer. Gastrointest Endosc. United States. 2009;69: 213-8.

37. Guo J, Li CQ, Li M, Zuo XL, Yu T, Liu JW, et al. Diagnostic value of probe-based confocal laser endomicroscopy and high-definition virtual chromoendoscopy in early esophageal squamous neoplasia. Gastrointest Endosc United States. 2015;81:1346-54.

38. Pech O, Rabenstein T, Manner H, Petrone MC, Pohl J, Vieth M, et al. Confocal laser endomicroscopy for in vivo diagnosis of early squamous cell carcinoma in the esophagus. Clin Gastroenterol Hepatol. W.B. Saunders; 2008 [cited 2019 Sep 1];6:89-94. Available from: https://www-sciencedirect-com.kuleuven.ezproxy. kuleuven.be/science/article/pii/S1542356507009743.

39. Mannath J, Ragunath K. Role of endoscopy in early oesophageal cancer. Nat Rev Gastroenterol Hepatol.England. 2016;13:720-30.

40. Picot J, Rose M, Cooper K, Pickett K, Lord J, Harris P, et al. Virtual chromoendoscopy for the real-time assessment of colorectal polyps in vivo: a systematic review and economic evaluation. Health Technol Assess (Rockv). 2017;21:1-307.

41. East JE, Vleugels JL, Roelandt P, Bhandari P, Bisschops R, Dekker E, et al. Advanced endoscopic imaging: European Society of Gastrointestinal Endoscopy (ESGE) Technology Review. Endoscopy. (C) Georg Thieme Verlag KG; 2016 [cited 2018 Dec 2];48:1029-45. Available from: http://www.thieme-connect. de/DOI/DOI?10.1055/s-0042-118087.

42. Singh R, Shahzad MA, Tam W, Goda K, Yu LHK, Fujishiro M, et al. Preliminary feasibility study using a novel narrow-band imaging system with dual focus magnification capability in Barrett's esophagus: is the time ripe to abandon random biopsies? Dig Endosc. 2013 [cited 2019 Jul 27];25:151-6. Available from: http://www.ncbi.nlm.nih.gov/pubmed/23617668.

43. Lee SK. Usefulness of probe-based confocal laser endomicroscopy for esophageal squamous cell neoplasm. Clin Endosc. 2019 [cited 2019 Nov 10];52:91-2. Available from: http://www.ncbi.nlm.nih. gov/pubmed/30909686.

44. Muto M, Hironaka S, Nakane M, Boku N, Ohtsu A, Yoshida S. Association of multiple Lugol-voiding lesions with synchronous and metachronous esophageal squamous cell carcinoma in patients with head and neck cancer. Gastrointest Endosc. United States. 2002;56:517-21.

45. Ishihara R, Takeuchi Y, Chatani R, Kidu T, Inoue T, Hanaoka N, etal.Prospectiveevaluation of narrow-bandimaging endoscopy for screening of esophageal squamous mucosalhigh-gradeneoplasiain experienced and less experienced endoscopists. Dis Esophagus United States. 2010;23:480-6.

Publisher's Note Springer Nature remains neutral with regard tojurisdictional claims in published maps and institutional affiliations. 